\title{
Bone Marrow Biopsy
}

National Cancer Institute

\section{Source}

National Cancer Institute. Bone Marrow Biopsy. NCI Thesaurus. Code C15193.

A biopsy involving the removal of a core tissue containing bone spicules and hematopoietic elements embedded in the marrow stroma. This procedure is done in the hip area mainly for the diagnosis and evaluation of neoplastic and non-neoplastic hematopoietic disorders (e.g. anemias, leukemias, lymphomas) and the evaluation of the spread of solid tumors (e.g. carcinomas, sarcomas) and lymphomas for therapeutic purposes. 